\title{
Disruption of the Coniothyrium minitans PIF1 DNA helicase gene impairs growth and capacity for sclerotial mycoparasitism
}

Correspondence

Michael P. Challen

mike.challen@warwick.ac.uk

Received 22 January 2008

Revised 13 March 2008

Accepted 17 March 2008

\author{
Christopher W. Rogers, Michael P. Challen, \\ Sreenivasaprasad Muthumeenakshi, Surapareddy Sreenivasaprasad \\ and John M. Whipps
}

Warwick HRI, University of Warwick, Wellesbourne, Warwickshire CV35 9EF, UK

\section{INTRODUCTION}

Coniothyrium minitans is a sclerotial mycoparasite of the plant-pathogenic fungus Sclerotinia sclerotiorum, which can infect over 400 plant species and causes severe economic loss in a wide variety of crops (Boland \& Hall, 1994; Budge et al., 1995; Escande et al., 2002; Huang et al., 2000; Willetts \& Wong, 1980). C. minitans is an effective biocontrol agent against Sclerotinia, in both field and glasshouse (Budge \& Whipps, 2001; Li et al., 2003), that can achieve levels of disease control comparable to fungicide treatments (Budge \& Whipps, 2001; Gerlagh et al., 1996). However, when disease levels are high, $C$. minitans fails to provide adequate control (Budge et al., 1995; Jones et al., 1999). To improve the efficacy of $C$. minitans as a biocontrol agent, better understanding of the molecular mechanisms involved in the fungal-fungal interaction and sclerotial mycoparasitism is required.

Infection of sclerotia by $C$. minitans does not involve specialized infection structures such as appressoria (Huang \& Hoes, 1976), but begins when the highly melanized sclerotial rind is penetrated by hyphal tips intracellularly or

Abbreviations: mtDNA, mitochondrial DNA; NES, nuclear exportation signal; $\mathrm{Phl}{ }^{\mathrm{R}}$, phleomycin-resistant; REMl, restriction enzyme-mediated DNA integration; ROS, reactive oxygen species; SEM, scanning electron microscopy. through intercellular gaps (Whipps \& Gerlagh, 1992). C. minitans hyphae penetrate host cell walls through a combination of mechanical pressure and degradation by extracellular enzymes, such as $\beta$-glucanases and chitinases (Huang \& Kokko, 1987; Jones \& Watson, 1969; Jones et al., 1974). Hyphal growth through infected tissues is followed by lysis and almost complete disintegration of the host cell walls accompanied by production of numerous pycnidia of $C$. minitans both internally and on the surface of the sclerotia.

Molecular characterization of $C$. minitans mycoparasitism is limited. The cmg1 exo- $\beta$-1,3-glucanase gene was shown to be upregulated when the fungus was grown on media containing sclerotia as the sole carbon source (Giczey et al., 2001). Recently we have identified 251 unique transcripts of C. minitans upregulated during growth on sclerotia, and representing genes involved in diverse processes including signalling, detoxification and stress responses (Muthumeenakshi et al., 2007). The development of efficient and reliable transformation and insertional mutagenesis systems for C. minitans, both protoplastmediated and Agrobacterium-mediated (ATMT), are enabling functional characterization of key genes (Jones et al., 1999; Rogers et al., 2004). Similar approaches have been used to identify pathogenicity genes in other plantpathogenic filamentous fungi, including Magnaporthe 
grisea and Fusarium graminearum (Balhadère et al., 1999; Kahmann \& Basse, 1999; Seong et al., 2005).

We previously used both REMI (restriction enzymemediated DNA integration) and ATMT insertional mutagenesis approaches to generate a library of over $4000 \mathrm{C}$. minitans transformants, from which nine 'pathogenicity mutants', unable to colonize sclerotia, were identified (Rogers et al., 2004). By analysing REMI mutant R2427, we have identified a disrupted gene that shows high homology to the PIF1 DNA helicase gene of Saccharomyces cerevisiae (Foury \& Lahaye, 1987). Functional complementation of R2427 has been achieved using the wild-type C. minitans gene. In other fungi, PIF1 is involved in mitochondrial DNA (mtDNA) repair and recombination and has a role in the maintenance of mitochondria in response to oxidative stress (Doudican et al., 2005; Foury \& Lahaye, 1987). This paper describes molecular characterization of the $C$. minitans PIF1 gene and demonstrates its concomitant role in mycelial growth and sclerotial mycoparasitism.

\section{METHODS}

Strains, plasmids and culture maintenance. The wild-type $C$. minitans strain Conio (IMI 134523), C. minitans REMI mutant R2427 (Rogers et al., 2004) and the host Scl. sclerotiorum isolate SB (IMI 390053) were maintained on potato dextrose agar (PDA, Oxoid) at $20{ }^{\circ} \mathrm{C}$ unless otherwise stated, and where appropriate with the addition of hygromycin (Rogers et al., 2004). Sclerotia of Scl. sclerotiorum were produced from PDA cultures. The plasmid FHJS3 (a gift from F. H. J. Schuren, University of Groningen, The Netherlands) contains the ble gene, encoding a phleomycin-binding protein, from Streptoalloteichus hindustanus (Schuren \& Wessels, 1994) coupled with the Agaricus bisporus gpdII promoter (Harmsen et al., 1992) and Schizophyllum commune gpd terminator (Schuren \& Wessels, 1998).

Molecular analysis of transformants. C. minitans genomic DNA was extracted from powdered freeze-dried mycelia, grown statically in potato dextrose broth (PDB, Oxoid) for 10-14 days, using the DNeasy Plant Mini kit (Qiagen). Southern blot analysis was performed according to standard protocols (Sambrook et al., 1989). Genomic DNA for PCR screening was isolated from C. minitans mycelia scraped from the surface of 4-day-old PDA cultures using a Chelex mini-prep method (Challen et al., 2003).

Total RNA was extracted from powdered freeze-dried mycelia grown for 10 days in PDB using the RNAgents Total RNA Isolation System (Promega). Samples were DNase treated (Promega) and reversetranscriptase PCR (RT-PCR) analysis was performed using the Eurogentec RT-PCR core kit with the following primers: X4prb (CGA ATG AAC CAG CTC CAA GG), X4prbC (GCG CTT GCA GAG AAG ATC AC), PIFendC (CCC TTC TTC GCT TTG CTC TC), $\beta$-tubulin-1 (ACT TTC TCC GTC GTC CCT TC), $\beta$-tubulin-3 (GCT GGT GAG AGG AGC GAA TC).

Recovery of disrupted sequences from R2427. The REMI mutagenesis site was identified using plasmid rescue. Genomic DNA (approx. $3 \mu \mathrm{g}$ ) was restricted with $\mathrm{XbaI}$, which cuts once within the transforming pAN7-1 sequence (Punt et al., 1987), purified using a QIAquick PCR purification column (Qiagen), self-ligated and transformed into Escherichia coli DH5 $\alpha$ competent cells (Invitrogen). Selection of ampicillin-resistant colonies allowed recovery of the recombinants containing the REMI-disrupted genomic DNA.
A macro-arrayed cosmid-based genomic library of $C$. minitans (Muthumeenakshi et al., 2007) was probed using a radioactively labelled 396 bp portion of the recovered XbaI fragment. Sequencing was performed using the BigDye Terminator v3.1 Cycle Sequencing kit and a 373 sequencer (Applied Biosystems) at the Warwick HRI Genomics Resource Centre.

Sequence analysis. Homologous sequences were identified using online tools BLAST (NCBI) or FASTA (EBI). The SE Central software package (Clone Manager Professional Suite v.8, Scientific \& Educational Software) and GENSCAN (http://genes.mit.edu/) were used to identify restriction sites, ORFs and introns, and to translate putative genes. Predicted protein sequences were analysed for specific motifs using PROSITE (ExPASy) and the CBS Prediction Server (http:// www.cbs.dtu.dk). The CLUSTAL $w$ algorithm in the MEGALIGN module (Lasergene v.7, DNASTAR) was used to create multiple protein sequence alignments, and presented using GeneDoc (v.2.6.002) (Nicholas et al., 1997). Dendrograms were created based on protein sequences using MEGALIGN and the ProtDist algorithm from PHYLIP (version 3.5c) (Felsenstein, 1993), with bootstrap sampling $(n=1000)$.

Sequences for C. minitans PIF1 are deposited in the EMBL nucleotide sequence database under accession numbers AM778550 and AM778832.

Complementation of R2427. The 4597 bp genomic DNA region encompassing the C. minitans PIF1 gene was amplified from a cosmid template, using the Expand High Fidelity system (Roche) with primers PIFampl (TGG GTG TCA TGC CGT CTT TG) and PIFamplC (GCC GGT GTA GAT GTT GGA GA), cloned using pGEM T Easy (Promega) and sequenced to check for amplification errors. The resulting pGEM_PIF1 plasmid and pFHJS3 were cotransformed into mutant R2427 using protoplast-mediated transformation (Rogers et al., 2004). Phleomycin-resistant colonies were selected and tested for their ability to parasitise Scl. sclerotiorum sclerotia as described previously (Rogers et al., 2004). Transformants were screened by PCR and Southern hybridization to confirm the presence of wild-type PIF1.

Scanning electron microscopy (SEM). Sclerotia were inoculated with wild-type $C$. minitans or R2427 using a conidial suspension and placed on water agar plates at $20{ }^{\circ} \mathrm{C}$ for $0-30$ days. Sclerotia were attached to the sample holder using Leit-C (Neubauer) conductive carbon cement before transfer to the cold stage of the cryopreparation chamber $\left(-160\right.$ to $\left.-180{ }^{\circ} \mathrm{C}\right)$. Samples were sputtercoated with gold for $5 \mathrm{~min}(1.5 \mathrm{kV}$ and $1.5-2 \mathrm{~mA})$ and transferred to the SEM cold stage. Examination of the samples was performed at approx. $-180{ }^{\circ} \mathrm{C}$ using a $10 \mathrm{kV}$ accelerating voltage in the presence of an anti-contaminator.

\section{RESULTS}

\section{Phenotype of REMI mutant R2427}

Previous work had shown that the growth rate of R2427 on PDA $(1.13 \pm 0.16 \mathrm{~mm}$ per day) was substantially reduced compared to wild-type $(3.77 \pm 0.2 \mathrm{~mm}$ per day $)(P<0.001)$ and that R2427 does not infect live sclerotia of Scl. sclerotiorum (Rogers et al., 2004). The mycelial colony morphology of R2427 was also markedly altered (Fig. 1), although R2427 was still able to sporulate normally. SEM revealed that spores of R2427 could germinate and form sparse mycelial growth on the surface of live sclerotia (Fig. 1). However, no evidence of growth within the sclerotia was detected during three replicated mycoparasitism tests 


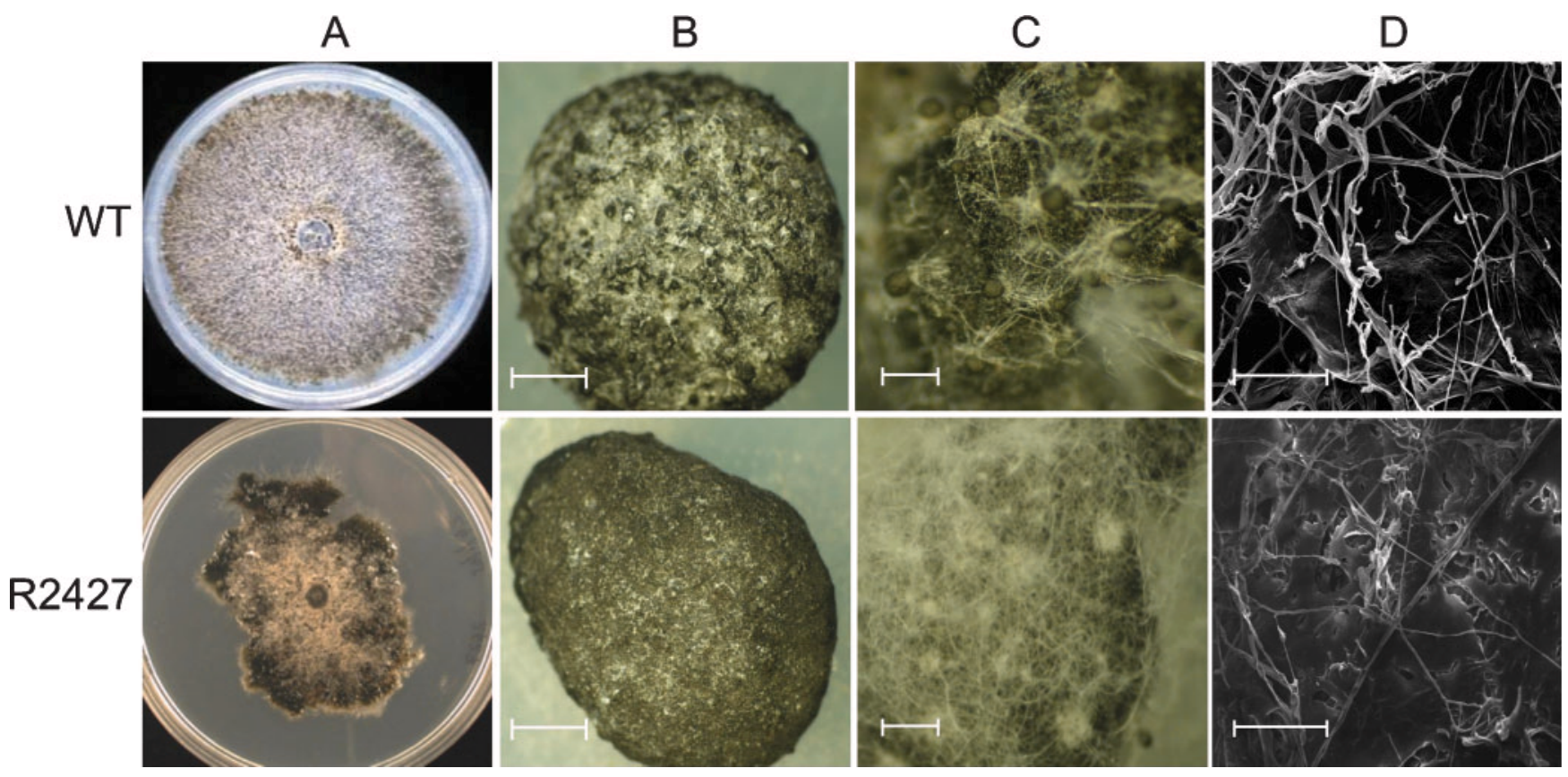

Fig. 1. Growth of C. minitans wild-type (WT) and the mutant R2427 on PDA and sclerotia of Scl. sclerotiorum. (A) 21 days' growth on PDA, single inoculation. R2427 has a reduced growth rate. (B) 18 days' growth on live sterile sclerotia, wild-type pycnidial initials visible, but no apparent growth of R2427. (C) 8 days' growth on autoclaved sclerotia. (D) SEM images of the sclerotial surface after 9 days' growth; sparse mycelial growth of R2427 compared to wild-type (bars, $50 \mu \mathrm{m}$ ).

of 20 sclerotia. R2427 readily colonized autoclaved sclerotia (Fig. 1), although pycnidial development occurred later than with wild-type C. minitans.

\section{R2427 contains a disrupted PIF1 gene}

Southern blot analysis of R2427 genomic DNA revealed tandem copies of pAN7-1 as a single integration at a HindIII recognition site, shown by a single hybridizing band in HindIII-digested R2427 DNA at approx. $6.8 \mathrm{~kb}$, the same size as the linear plasmid (Fig. 2).

Sequences upstream of the integration site were recovered by plasmid rescue using XbaI. A 2540 bp fragment of $C$. minitans DNA was sequenced and an internal 396 bp PCR product was used to detect the wild-type sequences. Comprehensive sequencing of the downstream flank of the integration site in R2427 was not performed, but PCR analysis showed that significant deletions had not occurred (data not shown) and that other ORFs were not disrupted by the REMI event.

Sequencing of a cosmid clone from the genomic library revealed a putative ORF of 2586 bp with a single 48 bp intron between positions 1148 and 1195 inclusive. Intron location was confirmed by sequencing two separate clones of the cDNA transcript (data not shown). The predicted 845 amino acid polypeptide (Fig. 3) had high similarity to the PIF1 genes of Aspergillus fumigatus (55\% identity),
A

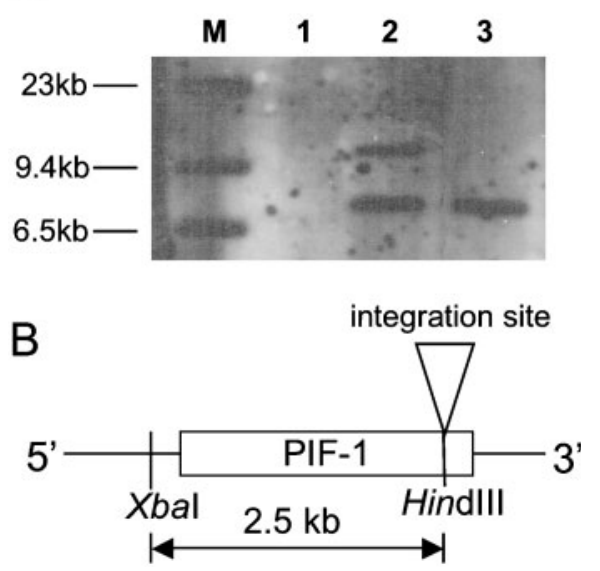

Fig. 2. Molecular analysis of the C. minitans PIF1 gene and the R2427 mutant. (A) Southern blot of DNA from wild-type and R2427. Hindlll- or Xbal-restricted DNA was probed with a 2401 bp DIG-labelled pAN7-1 Bg/ll-EcoRl fragment. Lanes: M, DIG-labelled DNA marker (Roche); 1, Hindlll-restricted wild-type DNA; 2, Xbal-restricted R2427 DNA; 3, Hindlll-restricted R2427 DNA. The smaller hybridizing band in lanes 2 and 3 corresponds to linearized pAN7-1; the larger hybridizing band in lane 2 corresponds to DNA fragment containing C. minitans disrupted sequence. (B) Schematic of C. minitans PIF1 gene region (not to scale); 2540 bp fragment cloned via plasmid rescue. 
Neurospora crassa (48\% identity) and Schizosaccharomyces pombe ( $47 \%$ identity). The C. minitans PIF1 ORF has two putative start codon methionine residues (Fig. 3; residues 1 and 53). The C. minitans PIF1 protein contains features consistent with DNA helicases: an ATPase domain (GSAGTGKS) at residues 380-387 and a putative DNAbinding domain (KGQAYVAL) at residues 780-787. Both domains are located in the conserved C-terminal region. BLASTP analysis revealed similarity of residues 312-814 to RecD ATP-dependent exoDNase (exonuclease V), alpha subunit (COG0507; $E=6 \times 10^{-29}$ ). This helicase superfamily 1 contains helicases involved in DNA replication, recombination and repair.

Analysis of the C. minitans PIF1 sequence showed a signal peptide predicted to target the protein to the mitochondria, with a cleavage site between residues 19 and 20. A nuclear exportation signal (NES) was predicted at position residue 759. Consistent with localization to the mitochondria, a prokaryotic membrane lipoprotein lipid attachment site was identified at residues 409-419. The REMI site in R2427 occurred within the codon of amino acid residue 781 and the highly conserved putative DNA-binding domain at the C-terminal end (Fig. 3).

In ProtDist analysis (Fig. 4), C. minitans PIF1 was most similar to the Phaeosphaeria nodorum protein and clustered with several Aspergillus species. PIF1 homologues from other filamentous fungi such as $M$. grisea and $N$. crassa formed a separate clade; the yeasts Cryptococcus neoformans and Saccharomyces cerevisiae were most distant.

\section{PIF1 disruption affects growth and sclerotial mycoparasitism}

The pGEM_PIF1 plasmid encompasses wild-type C. minitans PIF1, flanked by $\geqslant 1 \mathrm{~kb}$ of $5^{\prime}$ and $3^{\prime}$ sequences. Co-transformation of R2427 yielded 21 phleomycinresistant $\left(\mathrm{Phl}^{\mathrm{R}}\right)$ transformants from six independent transformation experiments, 16 of which contained wildtype PIF1 (Fig. 5). Restoration of wild-type colony growth rate and full sclerotial pathogenicity was observed in all 16 transformants containing wild-type PIF1 (type 1 and 3 transformants; Fig. 5). The five transformants that did not contain the wild-type gene were indistinguishable from R2427 in terms of growth and sclerotial pathogenicity. In six of the 16 complemented transformants the $h p h$ transgene could not be detected, suggesting that a homologous gene-replacement event had occurred at the PIF1 locus (type 3 transformants).

Hybridization of the 396 bp PIF1 probe to wild-type, R2427 and 14 randomly selected $\mathrm{Phl}^{\mathrm{R}} \mathrm{R} 2427$ transformants indicated that wild-type $C$. minitans contains a single copy of the PIF1 gene (data not shown). In R2427, the XbaIrestricted hybridizing band was larger (approx. $9.3 \mathrm{~kb}$ ) than in the wild-type (approx. $5.7 \mathrm{~kb}$ ), due to disruption of the ORF by the integrating plasmid. Complemented transformants (type 1 and 3) always contained the $5.7 \mathrm{~kb}$ band. Type 2 (non-complemented) transformants contained the $9.2 \mathrm{~kb}$ band but not the $5.7 \mathrm{~kb}$ band (Fig. 5B).

\section{PIF1 transcript analysis}

PIF1 transcripts were detectable in both wild-type and R2427 RNA samples from mycelia grown in PDB for 10 days (Fig. 6). Although 5' regions could be amplified using appropriate oligonucleotide primers, full-length PIF1 transcripts ( $3^{\prime}$ regions) could not be amplified from R2427 using RT-PCR (Fig. 6), suggesting that the transcript was truncated at the $3^{\prime}$ end. These observations were consistent with the integration of pAN7-1 after nucleotide 2394 in the PIF1 gene (Fig. 2B).

Virtual Northern analysis using randomly primed cDNAs indicated that levels of wild-type PIF1 expression were similar during both mycoparasitism and growth in PDB, and that transcript levels in R2427 grown in PDB were not altered (data not shown).

\section{DISCUSSION}

We have identified a putative DNA-helicase PIF1 gene from $C$. minitans that when disrupted results in reduced mycelial growth and an inability to mycoparasitize Scl. sclerotiorum sclerotia. Functional complementation of the PIF1 REMI mutant (R2427) and restoration of wild-type growth and mycoparasitic phenotypes was achieved using wild-type PIF1. We believe this is the first C. minitans gene shown to have a functional role in growth with concomitant effect on sclerotial mycoparasitism.

The predicted PIF1 protein ( $845 \mathrm{aa}$ ) has strong similarity with DNA-helicases from several other fungi and yeasts, including A. fumigatus, $N$. crassa and Sch. pombe. Interestingly, there was a high level of conservation in the C-terminal half of the proteins, in contrast to the $\mathrm{N}$ termini where the similarity was low between all species. Non-conserved N-terminal regions within PIF1 helicases may reflect specific properties such as differences in protein interactions, subcellular localization or additional enzymic activities (Bessler et al., 2001).

The role of PIF1 has been well characterized in Sac. cerevisiae and Sch. pombe, where it has been shown to be involved in repair and recombination of mitochondrial DNA (mtDNA) (Foury \& Lahaye, 1987), chromosomal DNA replication (Ivessa et al., 2000; Zhou et al., 2002), and maintenance of telomeres and rDNA (Ivessa et al., 2000; Schulz \& Zakian, 1994). PIF1 is not essential during the vegetative growth of Sac. cerevisiae, while the PIF1 homologue $(P f h 1)$ of Sch. pombe is essential, indicating different roles for PIF1 between species. The C. minitans PIF1 ORF has two putative start methionine codons separated by approx. 50 aa; similar architecture is observed in the yeast, human, A. fumigatus and N. crassa genes. The Sac. cerevisiae PIF1 is localized to both the nucleus and the mitochondria (Schulz \& Zakian, 1994; Zhou et al., 2000). 


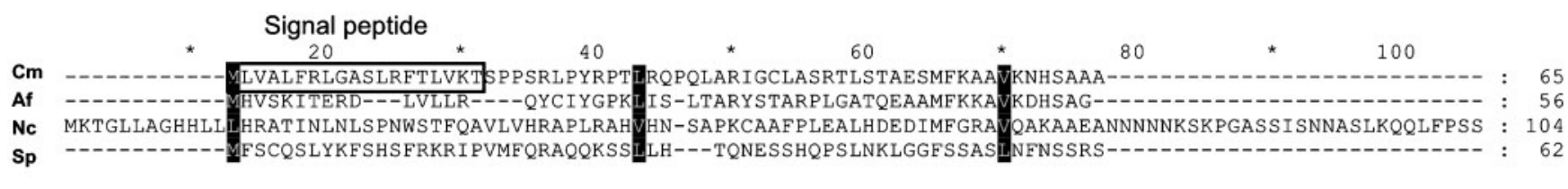

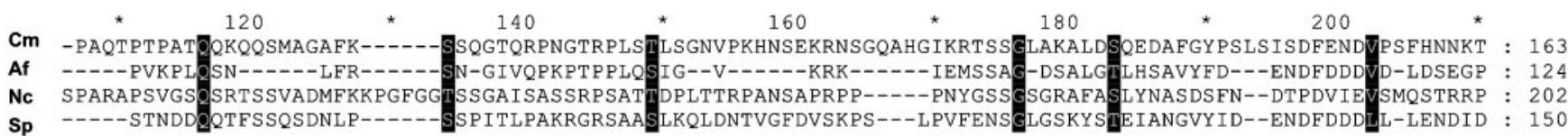

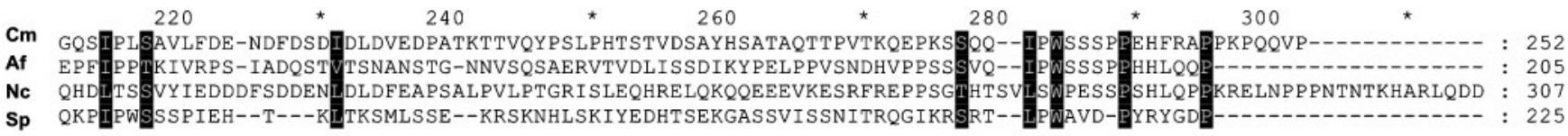
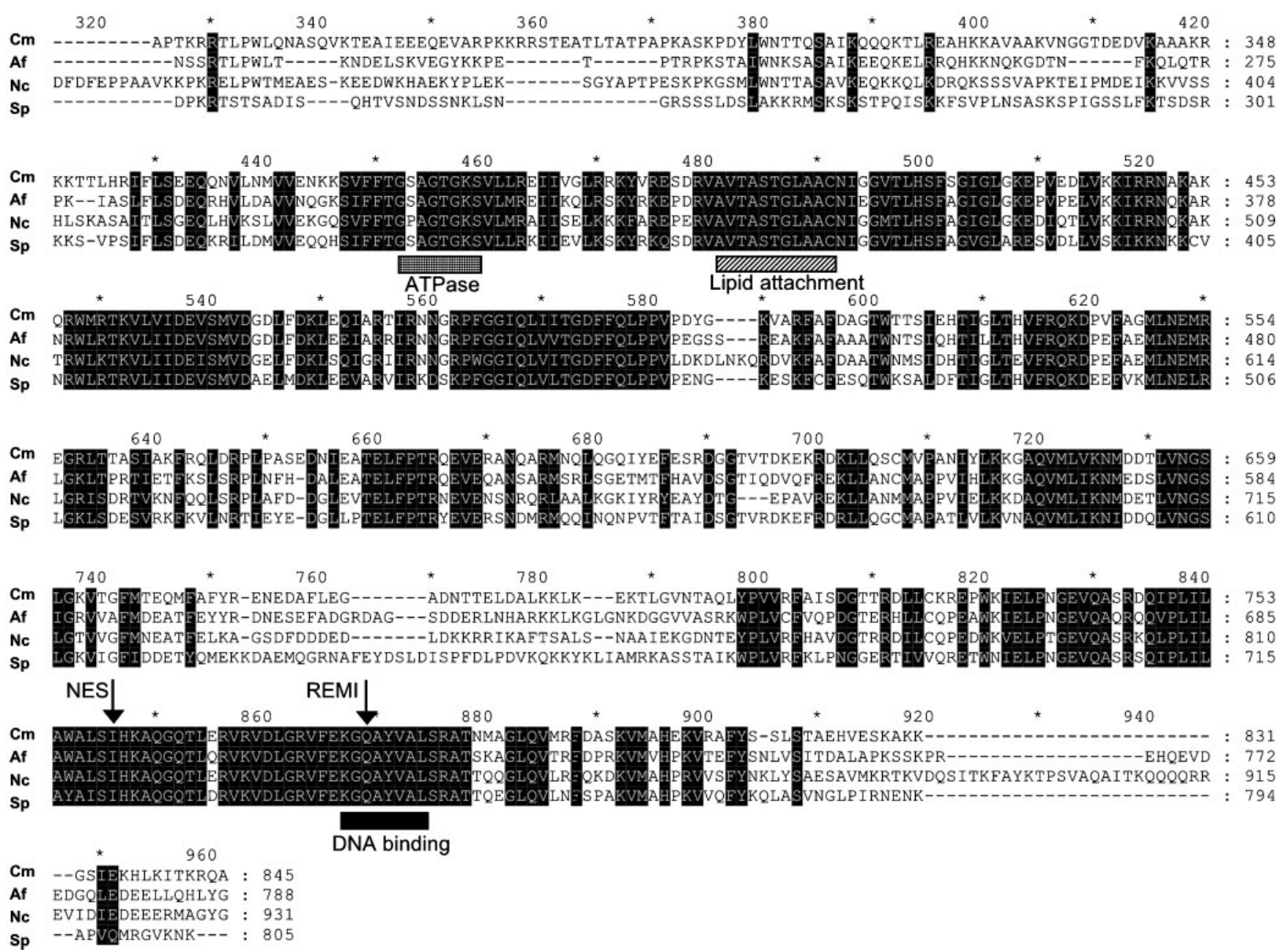

Fig. 3. Multiple sequence alignment of the PIF1 predicted proteins. Cm, Coniothyrium minitans; Af, Aspergillus fumigatus (accession no. XP_753317); Nc, Neurospora crassa (accession no. CAF05978); Sp, Schizosaccharomyces pombe (accession no. Q9UUA2). Shading represents $100 \%$ conserved residues. The predicted signal peptide is boxed; the ATP/ GTP-binding site motif A (P-loop) is underlined by a cross-hatched bar; the putative DNA-binding site is underlined with a solid bar. The diagonal striped bar denotes the location of the prokaryotic membrane lipoprotein lipid attachment site. Arrows indicate positions of the nuclear export signal (NES) and site of REMI integration (REMI). 


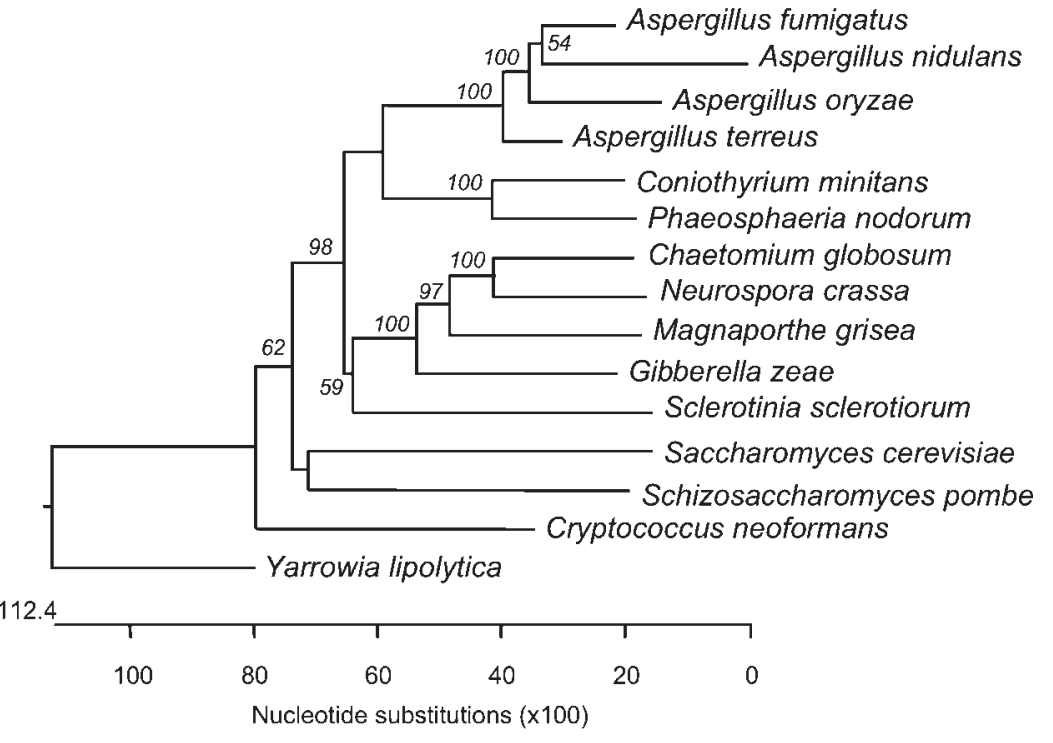

Fig. 4. Distance tree of PIF1 proteins. Alignment created using MEGALIGN and PHYLIP. Bootstrap support values $\geqslant 50 \%$ are in italics.
The nuclear and mitochondrial forms of PIF1 result from two length-disparate forms of the protein produced from different start codons (Schulz \& Zakian, 1994). In Sac. cerevisiae, mutation of the first methionine (PIF1-M1) resulted in mitochondrial instability, but telomere length and production remained as for wild-type. Conversely, mutation of the second methionine (PIF1-M2) affected telomere maintenance but not mitochondria (Schulz \& Zakian, 1994). Work with yeast and human PIF1 has also demonstrated that the ATP-binding domain plays an essential role in the function of the nuclear form, particularly in contributing to the maintenance of telomeres (Zhang et al., 2006; Zhou et al., 2000).

In R2427 the site of PIF1 disruption is located in the highly conserved C-terminal putative DNA-binding domain. Functional complementation of R2427 with wild-type PIF1 indicates that the DNA-binding domain and/or downstream sequences are responsible for the observed phenotype. It is noteworthy that while Sac. cerevisiae PIF1-

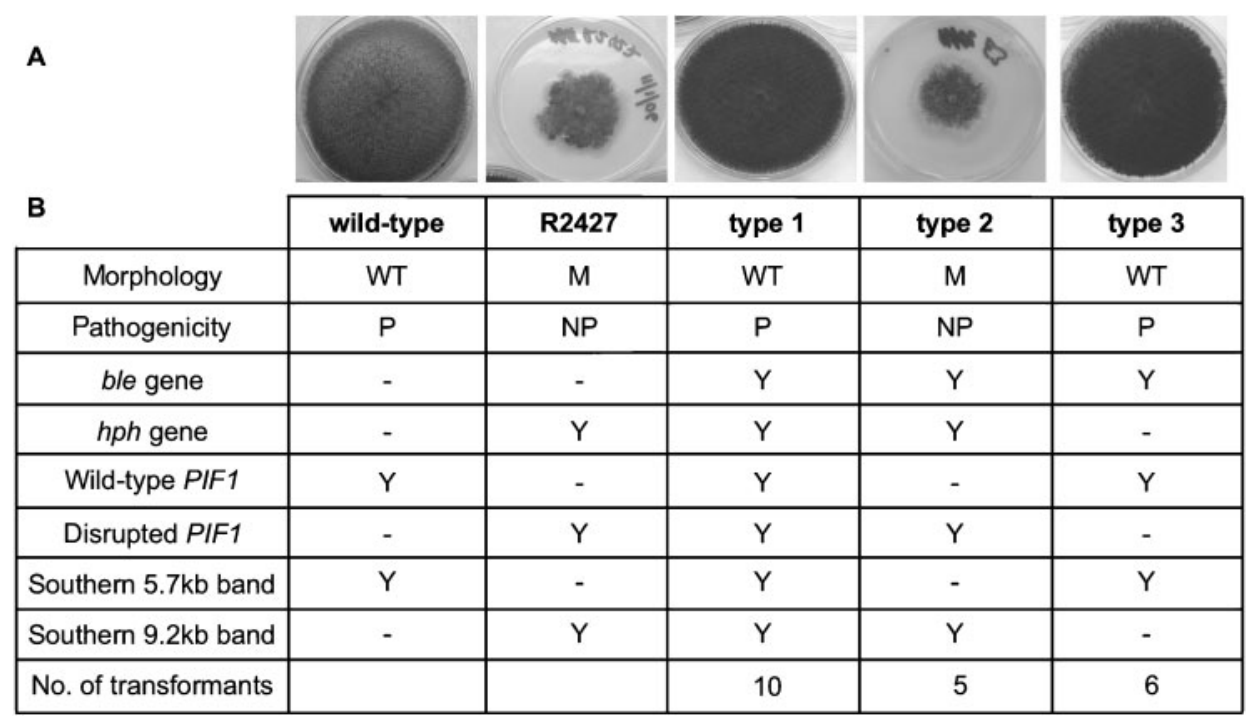

Fig. 5. Analysis of R2427 phleomycin-resistant transformants complemented with wild-type PIF1. (A) Wild-type, R2427 and $\mathrm{Phl}^{\mathrm{R}}$ transformants after 20 days growth on PDA. (B) Growth and pathogenicity of isolates, presence of genes after PCR analysis, summary of Southern analysis and transformant classification. Type 1 and 3 transformants are complemented, type 3 having lost the hph gene. Type 2 transformants do not contain wild-type PIF1. Y, gene present; -, gene absent; WT, wild-type morphology; M, mutant (R2427) morphology; P, mycoparasitic; NP, non-mycoparasitic. 


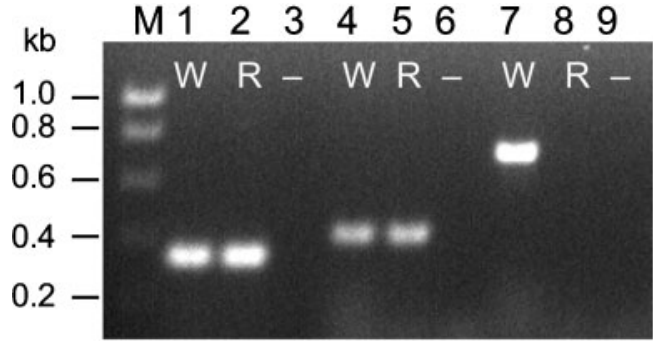

Fig. 6. RT-PCR analysis of C. minitans PIF1 after growth for 10 days in PDB. M, DNA marker (Bioline). Primers used for second strand synthesis: lanes $1-3, \beta$-tubulin-1 and $\beta$-tubulin-3; lanes 4-6, X4prb and X4prbC (PIF1 control region, $400 \mathrm{bp}$ ); lanes 7-9, X4prb and PIFendC (spanning PIF1 integration site in R2427). W, wild-type cDNA; R, R2427 cDNA; -, water control.

M2 mutant cells could grow as well as wild-type cells, PIF1M1 mutants (mitochondrial form) exhibited significantly higher proportions of petite cells (Schulz \& Zakian, 1994). The C. minitans R2427 mutant exhibits significantly reduced mycelial growth rates (Rogers et al., 2004). Aside from a possible nuclear role for the $C$. minitans PIF1, the above observations, coupled with the predicted N-terminal signal peptide for mitochondrial export, suggest that mitochondrial instability could result in the reduced growth and the loss of mycoparasitic capacity seen in R2427.

Mycoparasitism of C. minitans involves colonization of sclerotia (rather than antibiosis or competition for nutrients) and it would not be unexpected that inactivation of a gene critically impairing growth would also prejudice its mycoparasitic abilities. However, the loss of mycoparasitism in R2427 does not simply appear to be due to a reduction in growth rate. During insertional mutagenesis of C. minitans, several other transformants with reduced growth rates were found able to infect sclerotia over the same timeframe (Rogers, 2004). Our SEM studies showed that R2427 was able to germinate and grow on the sclerotial surface. The mutant was also able to colonize autoclaved sclerotia without difficulty and therefore was not impaired in its ability to mechanistically degrade and use dead sclerotial material as a nutrient source. Disruption of PIF1 therefore prevents C. minitans from penetrating and colonizing live sclerotia. However, PIF1 expression levels were not influenced by interaction with the host.

Complementation was achieved by co-transformation of the PIF1 gene with FHJS3, a plasmid containing the Streptoalloteichus hindustanus ble gene under the control of an Agaricus bisporus promoter, resulting in stable phleomycin-resistant transformants. This is believed to be the first reported use of the phleomycin resistance gene as a selectable marker in C. minitans, providing a useful tool for future molecular investigations. In a high proportion of the complemented transformants, the hygromycin resistance gene was replaced with the wild-type PIF1 gene, demonstrating that homologous recombination can occur at high frequency. Targeted gene knockouts are thus feasible in C. minitans and we have recently exploited this technology to disrupt other genes implicated in sclerotial mycoparasitism (C. W. Rogers and others, unpublished data).

Maintenance of the mitochondrial genome by PIF1 and repair of mtDNA following oxidative damage have been directly linked in yeast (O'Rourke et al., 2002; Zhou et al., 2002). In Sac. cerevisiae PIF1 plays a major role in maintaining mitochondrial integrity in response to oxidative stress (Doudican et al., 2005) and mutants showed a 30-fold increase in mitochondrial DNA mutagenesis (O'Rourke et al., 2002). There is also evidence that mtDNA is more susceptible than nuclear DNA (Yakes \& Van Houten, 1997) to oxidative damage by extracellular reactive oxygen species (ROS). Produced as unwanted byproducts of normal mitochondrial respiration during production of ATP through the oxidative phosphorylation pathway, ROS can damage cellular macromolecules, including mtDNA (Doudican et al., 2005). ROS can also originate exogenously, such as during the oxidative burst response to pathogen attack in plants (Apel \& Hirt, 2004). Such responses have been recently observed in a fungalfungal interaction between Ag. bisporus and Verticillium fungicola (Savoie \& Largeteau, 2004).

During mycoparasitism, C. minitans mtDNA may be exposed to increases in endogenous and/or exogenous ROS. Mycoparasitic growth and pressure-driven penetration of host cells could lead to endogenous ROS increases in mitochondria in C. minitans, while Scl. sclerotiorum may generate exogenous ROS in response to the mycoparasite. C. minitans PIF1 would therefore protect mtDNA from oxidative damage (and maintain mitochondrial stability) when exposed to increased ROS levels. R2427 with a dysfunctional PIF protein would suffer oxidative damage through its weakened repair mechanisms. This has been observed in Sac. cerevisiae, where PIF1 mutants display mitochondrial instability (Bessler et al., 2001). In turn, this would prevent the mutant from performing energydemanding processes, such as penetration and colonization of the heavily melanized sclerotia. This would explain our observations that R2427 is able to germinate and grow on the surface of the sclerotia but fails to penetrate and colonize internally.

The Scl. sclerotiorum host produces ROS as signalling molecules during sclerotial differentiation (Georgiou et al., 2001), but their presence in resting sclerotia or release during pathogen attack has not been reported. C. minitans expresses a wide range of genes related to ROS scavenging, oxidative stress response and DNA damage repair, including a histidine biosynthesis gene during sclerotial mycoparasitism (Muthumeenakshi et al., 2007). However, attempts to use histidine and proline as ROS scavengers (Chen \& Dickman, 2005; Son et al., 2005) with R2427 have 
hitherto proved inconclusive. Further work is required to provide a mechanistic understanding of the role of PIF1 DNA helicase in C. minitans development and sclerotial mycoparasitism.

\section{ACKNOWLEDGEMENTS}

This work was supported by grants from the BBSRC and EU project 2E-BCAs in Crops.

\section{REFERENCES}

Apel, K. \& Hirt, H. (2004). Reactive oxygen species: metabolism, oxidative stress, and signal transduction. Annu Rev Plant Biol 55, 373-399.

Balhadère, P. V., Foster, A. J. \& Talbot, N. J. (1999). Identification of pathogenicity mutants of the rice BLAST fungus Magnaporthe grisea by insertional mutagenesis. Mol Plant Microbe Interact 12, 129-142.

Bessler, J. B., Torres, J. Z. \& Zakian, V. A. (2001). The Piflp subfamily of helicases: region-specific DNA helicases? Trends Cell Biol 11, 60-65.

Boland, G. J. \& Hall, R. (1994). Index of plant hosts of Sclerotinia sclerotiorum. Can J Plant Pathol 16, 93-108.

Budge, S. P. \& Whipps, J. M. (2001). Potential for integrated control of Sclerotinia sclerotiorum in glasshouse lettuce using Coniothyrium minitans and reduced fungicide application. Phytopathology 91, 221-227.

Budge, S. P., McQuilken, M. P., Fenlon, J. S. \& Whipps, J. M. (1995). Use of Coniothyrium minitans and Gliocladium virens for biologicalcontrol of Sclerotinia sclerotiorum in glasshouse lettuce. Biol Control 5 , 513-522.

Challen, M. P., Kerrigan, R. W. \& Callac, P. (2003). A phylogenetic reconstruction and emendation of Agaricus section Duploannulatae. Mycologia 95, 61-73.

Chen, C. \& Dickman, M. B. (2005). Proline suppresses apoptosis in the fungal pathogen Colletotrichum trifolii. Proc Natl Acad Sci U S A 102, 3459-3464.

Doudican, N. A., Song, B. W., Shadel, G. S. \& Doetsch, P. W. (2005). Oxidative DNA damage causes mitochondrial genomic instability in Saccharomyces cerevisiae. Mol Cell Biol 25, 5196-5204.

Escande, A. R., Laich, F. S. \& Pedraza, M. V. (2002). Field testing of honeybee-dispersed Trichoderma spp. to manage sunflower head rot (Sclerotinia sclerotiorum). Plant Pathol 51, 346-351.

Felsenstein, J. (1993). PHYLIP (phylogeny inference package), version 3.5c. Distributed by the author. Department of Genome Sciences, University of Washington, Seattle, USA.

Foury, F. \& Lahaye, A. (1987). Cloning and sequencing of the Pif gene involved in repair and recombination of yeast mitochondrial-DNA. EMBO J 6, 1441-1449.

Georgiou, C. D., Tairis, N. \& Polycratis, A. (2001). Production of betacarotene by Sclerotinia sclerotiorum and its role in sclerotium differentiation. Mycol Res 105, 1110-1115.

Gerlagh, M., Whipps, J. M., Budge, S. P. \& Goossen van de Geijn, H. M. (1996). Efficiency of isolates of Coniothyrium minitans as mycoparasites of Sclerotinia sclerotiorum, Sclerotium cepivorum and Botrytis cinerea on tomato stem pieces. Eur J Plant Pathol 102, 787793.

Giczey, G., Kerenyi, Z., Fulop, L. \& Hornok, L. (2001). Expression of cmgl, an exo-beta-1,3-glucanase gene from Coniothyrium minitans, increases during sclerotial parasitism. Appl Environ Microbiol 67, 865-871.
Harmsen, M. C., Schuren, F. H. J., Moukha, S. M., van Zuilen, C. M., Punt, P. J. \& Wessels, J. G. H. (1992). Sequence analysis of the glyceraldehyde-3-phosphate dehydrogenase genes from the basidiomycetes Schizophyllum commune, Phanerochaete chrysosporium and Agaricus bisporus. Curr Genet 22, 447-454.

Huang, H. C. \& Hoes, J. A. (1976). Penetration and infection of Sclerotinia sclerotiorum by Coniothyrium minitans. Can J Bot 54, 406-410.

Huang, H. C. \& Kokko, E. G. (1987). Ultrastructure of hyperparasitism of Coniothyrium minitans on sclerotia of Sclerotinia sclerotiorum. Can $J$ Bot 65, 2483-2489.

Huang, H. C., Bremer, E., Hynes, R. K. \& Erickson, R. S. (2000). Foliar application of fungal biocontrol agents for the control of white mold of dry bean caused by Sclerotinia sclerotiorum. Biol Control 18, 270-276.

Ivessa, A. S., Zhou, J. Q. \& Zakian, V. A. (2000). The Saccharomyces Piflp DNA helicase and the highly related Rrm3p have opposite effects on replication fork progression in ribosomal DNA. Cell 100, 479-489.

Jones, D. \& Watson, D. (1969). Parasitism and lysis by soil fungi of Sclerotinia sclerotiorum (Lib.) de Bary, a phytopathogenic fungus. Nature 224, 287-288.

Jones, D., Gordon, A. H. \& Bacon, J. S. D. (1974). Co-operative action by endo- and exo- $\beta-(1 \rightarrow 3)$-glucanases from parasitic fungi in degradation of cell-wall glucans of Sclerotinia sclerotiorum (Lib) Bary. Biochem J 140, 47-55.

Jones, E., Carpenter, M., Fong, D., Goldstein, A., Thrush, A., Crowhurst, R. \& Stewart, A. (1999). Co-transformation of the sclerotial mycoparasite Coniothyrium minitans with hygromycin B resistance and beta-glucuronidase markers. Mycol Res 103, 929-937.

Kahmann, R. \& Basse, C. (1999). REMI (Restriction Enzyme Mediated Integration) and its impact on the isolation of pathogenicity genes in fungi attacking plants. Eur J Plant Pathol 105, 221-229.

Li, G. Q., Huang, H. C. \& Acharya, S. N. (2003). Importance of pollen and senescent petals in the suppression of alfalfa blossom blight (Sclerotinia sclerotiorum) by Coniothyrium minitans. Biocontrol Sci Technol 13, 495-505.

Muthumeenakshi, S., Sreenivasaprasad, S., Rogers, C. W., Challen, M. P. \& Whipps, J. M. (2007). Analysis of cDNA transcripts from Coniothyrium minitans reveals a diverse array of genes involved in key processes during sclerotial mycoparasitism. Fungal Genet Biol 44, 1262-1284.

Nicholas, K. B., Nicholas, H. B. J. \& Deerfield, D. W. (1997). GeneDoc: analysis and visualization of genetic variation. EMBNEWS 4, 14.

O'Rourke, T. W., Doudican, N. A., Mackereth, M. D., Doetsch, P. W. \& Shadel, G. S. (2002). Mitochondrial dysfunction due to oxidative mitochondrial DNA damage is reduced through cooperative actions of diverse proteins. Mol Cell Biol 22, 4086-4093.

Punt, P. J., Oliver, R. P., Dingemanse, M. A., Pouwels, P. H. \& van den Hondel, C. A. M. J. J. (1987). Transformation of Aspergillus based on the hygromycin B resistance marker from Escherichia coli. Gene 56, 117-124.

Rogers, C. W. (2004). Molecular approaches towards the identification of pathogenicity genes in the mycoparasite Coniothyrium minitans. $\mathrm{PhD}$ thesis, University of Birmingham, 245pp.

Rogers, C. W., Challen, M. P., Green, J. R. \& Whipps, J. M. (2004). Use of REMI and Agrobacterium-mediated transformation to identify pathogenicity mutants of the biocontrol fungus, Coniothyrium minitans. FEMS Microbiol Lett 241, 207-214.

Sambrook, J., Fritsch, E. F. \& Maniatis, T. (1989). Molecular Cloning: a Laboratory Manual, 2nd edn. Cold Spring Harbor, NY: Cold Spring Harbor Laboratory. 
Savoie, J.-M. \& Largeteau, M. L. (2004). Hydrogen peroxide concentrations detected in Agaricus bisporus sporocarps and relation with their susceptibility to the pathogen Verticillium fungicola. FEMS Microbiol Lett 237, 311-315.

Schulz, V. P. \& Zakian, V. A. (1994). The Saccharomyces Pif1 DNA helicase inhibits telomere elongation and de-novo telomere formation. Cell 76, 145-155.

Schuren, F. H. J. \& Wessels, J. G. H. (1994). Highly efficient transformation of the homobasidiomycete Schizophyllum commune to phleomycin resistance. Curr Genet 26, 179-183.

Schuren, F. H. J. \& Wessels, J. G. H. (1998). Expression of heterologous genes in Schizophyllum commune is often hampered by the formation of truncated transcripts. Curr Genet 33, 151-156.

Seong, K., Hou, Z. M., Tracy, M., Kistler, H. C. \& Xu, J. R. (2005). Random insertional mutagenesis identifies genes associated with virulence in the wheat scab fungus Fusarium graminearum. Phytopathology 95, 744-750.

Son, D. O., Satsu, H. \& Shimizu, M. (2005). Histidine inhibits oxidative stress- and TNF-alpha-induced interleukin-8 secretion in intestinal epithelial cells. FEBS Lett 579, 4671-4677.
Whipps, J. M. \& Gerlagh, M. (1992). Biology of Coniothyrium minitans and its potential for use in disease biocontrol. Mycol Res 96, 897-907.

Willetts, H. J. \& Wong, J. A. L. (1980). The biology of Sclerotinia sclerotiorum, S. trifoliorum, and S. minor with emphasis on specific nomenclature. Bot Rev 46, 101-165.

Yakes, F. M. \& Van Houten, B. (1997). Mitochondrial DNA damage is more extensive and persists longer than nuclear DNA damage in human cells following oxidative stress. Proc Natl Acad Sci U S A 94, 514-519.

Zhang, D. H., Zhou, B., Huang, Y., Xu, L. X. \& Zhou, J. Q. (2006). The human Pifl helicase, a potential Escherichia coli RecD homologue, inhibits telomerase activity. Nucleic Acids Res 34, 1393-1404.

Zhou, J. Q., Monson, E. K., Teng, S. C., Schulz, V. P. \& Zakian, V. A. (2000). Piflp helicase, a catalytic inhibitor of telomerase in yeast. Science 289, 771-774.

Zhou, J.-Q., Qi, H., Schulz, V. P., Mateyak, M. K., Monson, E. K. \& Zakian, V. A. (2002). Schizosaccharomyces pombe pfh1+ encodes an essential 5' to $3^{\prime}$ DNA helicase that is a member of the PIF1 subfamily of DNA helicases. Mol Biol Cell 13, 2180-2191.

Edited by: H. A. B. Wösten 